\title{
Formation of Autapse Connected to Neuron and Its Biological Function
}

\author{
Chunni Wang, ${ }^{1}$ Shengli Guo, ${ }^{1}$ Ying Xu, ${ }^{1}$ Jun Ma, ${ }^{1,2}$ Jun Tang, ${ }^{3}$ \\ Faris Alzahrani, ${ }^{2}$ and Aatef Hobiny ${ }^{2}$ \\ ${ }^{1}$ Department of Physics, Lanzhou University of Technology, Lanzhou 730050, China \\ ${ }^{2}$ NAAM-Research Group, Department of Mathematics, Faculty of Science, King Abdulaziz University, \\ P.O. Box 80203, Jeddah 21589, Saudi Arabia \\ ${ }^{3}$ College of science, China University of Mining and Technology, Xuzhou 221116, China
}

Correspondence should be addressed to Jun Ma; hyperchaos@163.com

Received 9 September 2016; Revised 4 January 2017; Accepted 17 January 2017; Published 28 February 2017

Academic Editor: Mattia Frasca

Copyright (C) 2017 Chunni Wang et al. This is an open access article distributed under the Creative Commons Attribution License, which permits unrestricted use, distribution, and reproduction in any medium, provided the original work is properly cited.

\begin{abstract}
Autapse is a specific synapse connected to the neuron via close loop, and its functional adjusting is described by applying timedelayed feedback on the membrane potential of the neuron. This paper discussed the possible formation mechanism and biological function of autapse connection on neurons. We believe that the formation and growth of autapse connected to neuron can be associated with injury on axon and blocking in signal transmission; thus auxiliary loop is developed to form an autapse. When autapse is set up, it can propagate the signals and change the modes of electrical activities under self-adaption. Based on the cable neuron model, the injury on axon is generated by poisoning and blocking in ion channels (of sodium); thus the conductance of ion channels are changed to form injury-associated defects. Furthermore, auxiliary loop with time delay is designed to restore and enhance signal propagation by setting different time delays and feedback gains. The numerical studies confirmed that appropriate time delay and feedback gain in electric or chemical autapse can help signal (or wave generated by external forcing) propagation across the blocked area. As a result, formation of autapse could be dependent on the injury of neuron and further enhances the self-adaption to external stimuli.
\end{abstract}

\section{Introduction}

Neuronal models [1-6] are helpful to understand the main dynamical properties in neuronal activities, and most of the simplified versions have been developed from the biological Hodgkin-Huxley neuron [1] model with the effect of ion channels being considered. Despite the continuous dynamical neuron model, Ibarz et al. [7] argued that map-based neuron model could be also effective to describe the main properties of electrical activities. In fact, the astrocyte [8] also plays important role in regulating the electrical activities of neuron; as a result, neuron coupled astrocyte models [9-12] have been proposed to study the seizure-like discharges (SDs) or seizure-like firings (SFs) in neurons. Furthermore, the dependence of electrophysiological activities on energy consumption and metabolism is discussed, and Wang et al. [13-15] proposed an energy model to investigate the mode transition associated with energy supply. It is believed that signal transmission between neurons can be realized by chemical and/or electrical synapse coupling, while Volman et al. [16] argued that the role of gap junction connection between neurons could be much complex during the emergence of epileptic seizures. Ion channels [17] are embedded into the membrane of neuron, and their stochastic on and off can generate channel noise; thus the electrical activities of neurons can be changed. As a result, ion channels are blocked to detect the transition of electrical activities in neuron and neuronal network [18-23]. There are some physical factors that should be mentioned during the fluctuation of extracellular and intracellular ion concentration, which can generate electromagnetic induction among cells; thus the membrane potentials can be adjusted. Therefore, the authors of $[24,25]$ suggested 
that magnetic flux can be used to model the electromagnetic induction on neuronal activities and confirmed that multiple modes of electrical activities can be induced by changing one bifurcation parameter. The realistic neuronal system contains a large number of neurons, and it is believed that network could be helpful to investigate the collective behaviors of neurons [26-30].

By now, oscillator-like neuron models have been improved greatly so that more biological factors can be considered. For example, autapse [31, 32], a specific synapse connected to its body via a close loop, and this type of feedback with time delay can change the electrical activities of neurons [33-35]. Indeed, experimental evidence confirmed the occurrence of autapse [36-40]. It is found that neuron can give sensitive response to autapse driving while chemical autapse can change the electrical activities of neuron in slight way [41-45]. For example, neuron can give rapid response in case of electrical autapse connection, and appropriate feedback type (positive or negative feedback in autapse) can change the excitability of neuron. In case of positive feedback in autapse, neuron can be excited while negative feedback in autapse can calm down the electrical activities in neurons. It is the electrical autapse compared to the chemical autapse that can be more effective to change the mode in electrical activities greatly. For a brief review, please find the survey in [45] and some reference therein. In the case of synchronization of network [46, 47], autapse driving can often enhance the synchronization with appropriated feedback gain and time delay being used, where the collective behaviors of network can be modulated like a pacemaker generated by autapse. Furthermore, appropriate distribution and driving of autapse in the network can regulate and block the collective behaviors like a pacemaker or even a defect that can enhance the pattern formation and synchronization in the network [48-50].

As mentioned in [51], autapse is an unusual type of synapse generated by a neuron on itself and autapse connection can play complex biological function by changing the electrical activities in neuron and neuronal networks [26]. That is, emergence of defects due to negative feedback can block the wave propagation, while regularity is enhanced by pacemaker generated by positive feedback in autapse. Unfortunately, it keeps open on how an autapse can be formed. Indeed, monitoring such growth of autapse is technically challenging due to the requirement for precise capture and long-term analysis of single neuron in three dimensions (3D). The authors of [51] presented a simple two-step photolithography method to efficiently capture single cells in microscale gelatin methacrylate hydrogel rings and culture single neurons; these results demonstrated that neural axons grew and consequently formed axonal circles, indicating that our method could be an enabling tool to analyze axonal development and autapse formation. In this paper, we argue that the formation of autapse connected to neuron could be associated with injury on axon that signal propagation is blocked; thus another auxiliary loop is developed to help signal transmission and self-adjusting on mode selection of electrical activities.

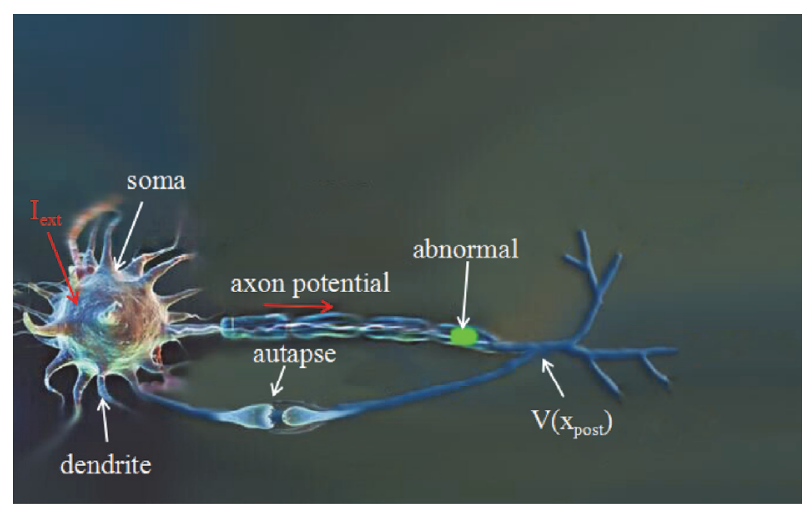

FIGURE 1: Schematic diagram of injured neuron driven by autapse: action potential is propagated along the axon and the local kinetics of electrical activities will be described by Hodgkin-Huxley neuron model. Abnormal area on the axon is generated by blocking channels of sodium or potassium; thus the conductance is used as bifurcation parameter. When neuron (axon) is injured, synapse is triggered to form auxiliary loop; thus autapse can be formed.

\section{Autapse Formation Mechanism and Model Description}

The ability to monitor axonal growth of single neuron and autapse formation in 3D may provide fundamental information relating to many cellular processes, such as axonal development, synaptic plasticity, and neural signal transmission. In this section, the possible physical mechanism for autapse growth will be discussed. According to Figure 1, appropriate external forcing can generate possible response on the neuron and action potential can be triggered to propagate along the axon. However, signal transmission can be terminated or blocked when the axon is injured, for example, poisoning in ion channels or heterogeneity on local area of the axon. As a result, neuron can develop new loop or secondary loop to help signal transmission. For the oscillator-like neuron model developed from the Hindmarsh-Rose neuron [52, 53] and Hodgkin-Huxley neuron model [54, 55], the electrical autapse current and chemical autapse current are, respectively, described as follows:

$$
\begin{aligned}
& I_{\text {aut }_{e}}=g_{e}[x(t-\tau)-x(t)] \\
& I_{\text {aut }_{c}}=-\frac{g_{c}\left[x(t)-V_{\text {syn }}\right]}{\left[1+e^{-\lambda\left[x\left(t-\tau^{\prime}\right)-\theta\right]}\right]},
\end{aligned}
$$

where $g_{e}$ and $g_{c}$ are the gains for electrical and chemical autapse, respectively. $x$ is membrane potential of neuron described in HR neuron model. $\tau$ is time delay (intrinsic response time delay, and it is different form the propagation time delay due to finite propagation speed of signals between neurons) in electrical autapse and $\tau^{\prime}$ is the time delay in chemical autapse. $x(t)$ is the sampled membrane potential of neuron, $V_{\text {syn }}$ is reversal potential in synapse and is used to discern excitatory synapse at $V_{\text {syn }}=2$ and for inhibitory synapse at $V_{\text {syn }}=-2$. The ratio parameter $\lambda$ and threshold $\theta$ for synapse are often selected as $\lambda=10$ and $\theta=-0.25$ in previous 
investigations, respectively. In fact, the anatomical structure of neuron should be considered in case of signal transmission in neuron; for example, the signal propagation along axon should be investigated in detail. Our cable-injured neuron model can be illustrated in Figure 1.

The model is set up on cable neuron model, auxiliary loop is developed when the axon is injured in local area, and new dendrites can be triggered close to the injured area on the axon and then are connected to other dendrites connected to the soma. As a result, important signal can be transmitted along the auxiliary loop called autapse under positive feedback. The feedback gain and time delay can be selected to recover the original signal propagation. The second biological function of autapse is that electrical modes of neuronal activities can be selected under appropriate negative feedback in autapse. That is to say, negative feedback in autapse can play similar function as inhibitory synapse. For further dynamical investigation and discussion, our proposed neuron model driven by autapse can be expressed as follows:

$$
C \frac{d V(x)}{d t}=f(V(x), p)+D \frac{d^{2} V}{d x^{2}}+I_{\text {aut }},
$$

where $C$ and $D$ denote the membrane capacitance and the diffusive coefficient, respectively. $V(x)$ represents membrane potential at position $x$ and $I_{\text {aut }}$ is the autapse current. The function $f(V(x), p)$ describes the local kinetics of electrical activities, $p=\left\{G_{\max }^{\mathrm{K}}, G_{\max }^{\mathrm{Na}}, G_{L}\right\}$ is the parameters such as conductance for ion channels, and $G_{\max }^{\mathrm{K}}, G_{\max }^{\mathrm{Na}}, G_{L}$ is the maximal conductance for potassium channels, sodium channels, and leakage current associated with chloridion channels. For simplicity, local kinetics of the cable neuron model can be described by Hodgkin-Huxley equations as follows:

$$
\begin{aligned}
f(V(x), p)= & G_{\mathrm{Na}} m^{3} h\left(V_{\mathrm{Na}}-V(x)\right) \\
& +G_{\mathrm{K}} n^{4}\left(V_{\mathrm{K}}-V(x)\right) \\
& +G_{L}\left(V_{L}-V(x)\right) \\
\frac{d y}{d t}= & \alpha_{y}(V)(1-y)+\beta_{y}(V) y,
\end{aligned}
$$

$$
y=m, n, h
$$

where $V_{\mathrm{Na}}, V_{\mathrm{K}}$, and $V_{L}$ are the reversal potentials for the sodium, potassium, and leakage currents, respectively. It is suggested that the gating dynamics of each ion channel are assumed to be governed by four independent gates, each of which can switch between an open and a closed conformation. The gating variables $n, m$, and $h$ describe the mean ratios of the open gates of the working channels, and the factors $n^{4}$ and $m^{3} h$ are the mean portions of the open ion channels within the membrane patch. The voltage-dependent opening and closing rates $\alpha_{y}(V), \beta_{y}(V)$, and $y=m, n, h$ are defined in [56-58]. The abnormal area on the axon could be formed due to channels blocking; for example, the potassium or sodium ion channels can be, respectively, blocked or disabled by adding cell toxins such as tetraethylammonium (TEA) and/or tetrodotoxin (TTX) in experiments. As reported in [56], the poisoning of sodium channels causes only a small, practically negligible variation of the resting voltage; a reduction in the number of working potassium channels changes dramatically the qualitative behavior of the spiking activity. In fact, neuron and its axon can be regarded as injured when the ion channels of sodium or potassium are blocked; thus the conductance of channels is changed in parameter values. As a result, the conductance of ion channels in the abnormal area (heterogeneity) can be defined as follows:

$$
\begin{aligned}
G_{\mathrm{Na}} & =G_{\max }^{\mathrm{Na}} \chi_{\mathrm{Na}} m^{3} h, \\
G_{\mathrm{K}} & =G_{\max }^{\mathrm{K}} \chi_{\mathrm{K}} n^{4},
\end{aligned}
$$

where the active ratios $\chi_{\mathrm{Na}}$ and $\chi_{\mathrm{K}}$ are the fractions of working channels, that is, nonblocked ion channels, to the overall number of potassium $\left(N_{\mathrm{K}}, N_{\mathrm{Na}}\right)$ ion channels, respectively.

According to Figure 1, the electrical and chemical autapse current on the feedback loop are defined by

$$
\begin{aligned}
I_{\mathrm{aut}_{e}} & =g_{e}\left[V\left(t-\tau, x_{\text {post }}\right)-V(t, x)\right] \\
I_{\text {aut }_{c}} & =-\frac{g_{c}\left[V(t, x)-V_{\text {syn }}\right]}{\left[1+e^{-\lambda\left[V\left(t-\tau^{\prime}, x_{\text {post }}\right)-\theta\right]}\right]},
\end{aligned}
$$

where the axon orientation (or direction) is used for $x$-axis for mathematical description, the variable $x_{\text {post }}$ represents the beginning point to follow the injured area where autapse can be developed, and the parameters in (5) are described the same as shown in (1). That is to say, when normal signal propagation is blocked along the axon, auxiliary loop is required to form autapse connection. To ensure effective formation of autapse, some dendrites can be developed from the soma to connect the synapse developed from the area close to injured area, and then autapse can be formed. Therefore, the autapse current can be written as follows:

$$
\begin{aligned}
I_{\text {aut }_{e}} & =g_{e}\left[V\left(t-\tau, x_{\text {post }}\right)-V(t, 0)\right] \\
I_{\text {aut }_{c}} & =-\frac{g_{c}\left[V(t, 0)-V_{\text {syn }}\right]}{\left[1+e^{-\lambda\left[V\left(t-\tau^{\prime}, x_{\text {post }}\right)-\theta\right]}\right]} .
\end{aligned}
$$

That is to say, a new autapse can be developed from the soma and the injured area synchronously. As a result, blocked signals can be transmitted along the autapse under positive feedback with appropriate feedback gain and time delay being used. When the autapse is set up, negative feedback in the autapse can be helpful for the selection of modes for electrical activities by applying appropriate feedback gain and time delay. The formation of autapse also develops the selfadaption of neuron; thus appropriate response can be selected during the signal propagation in neuron and between neurons.

\section{Results, Discussion, and Open Problems}

The formation of autapse is independent of the feedback loop, and the time delay in the autapse could accounts for the beginning and end of the autapse formation. When 


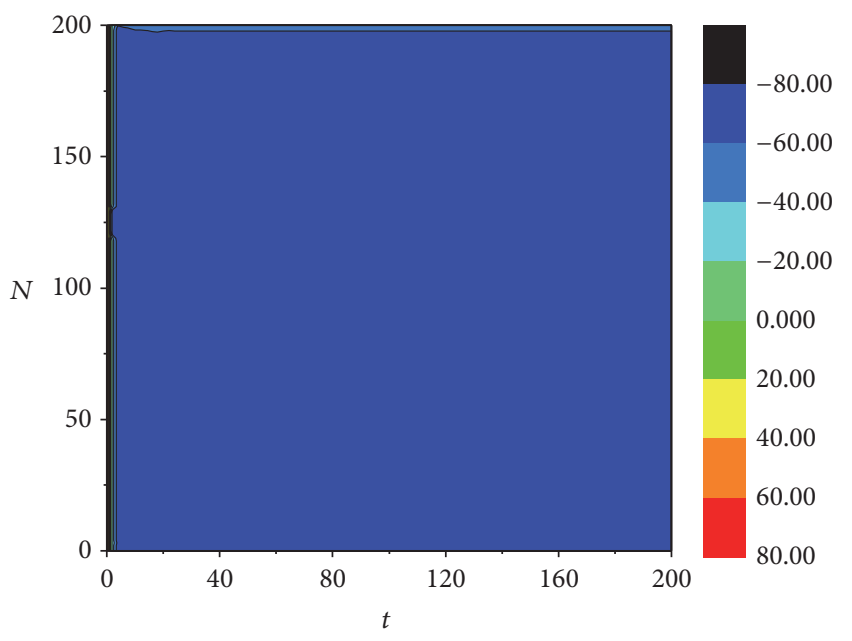

(a)

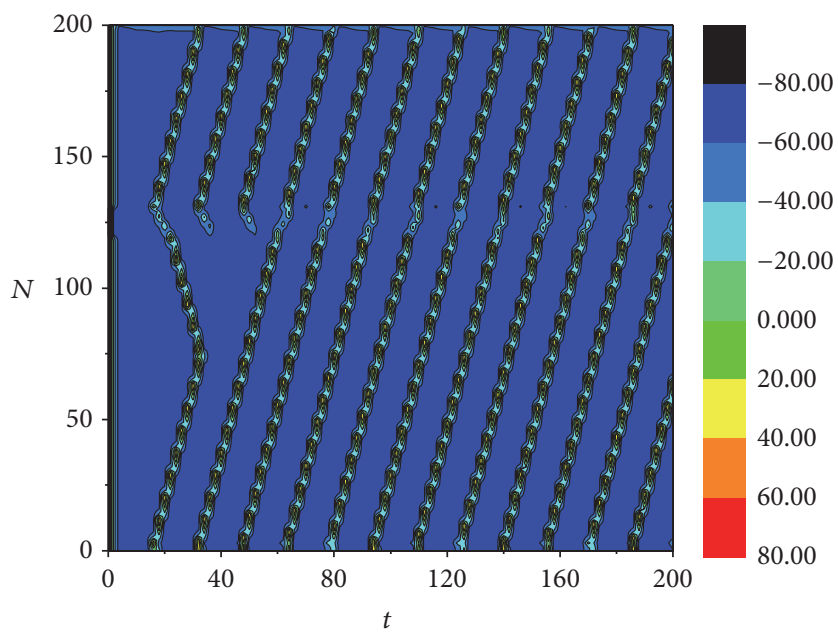

(c)

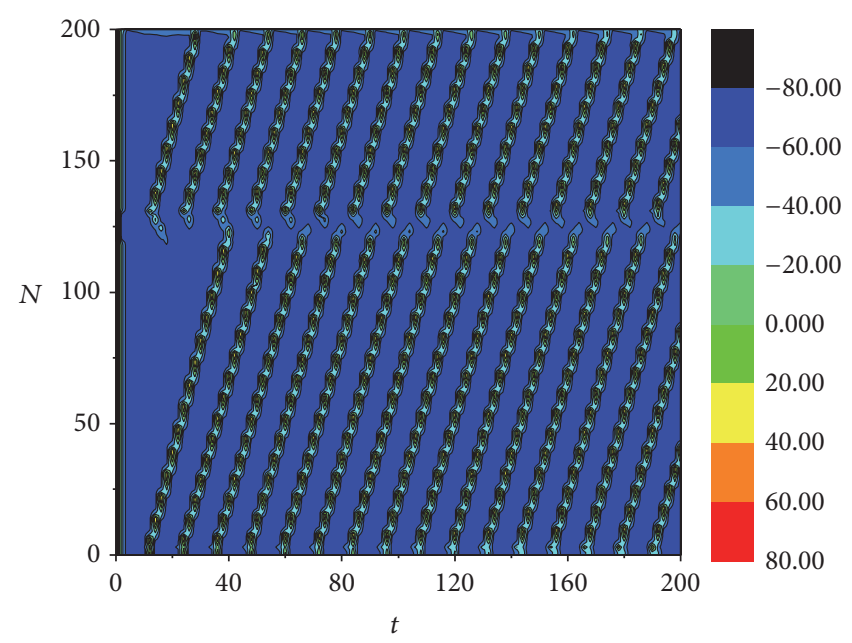

(b)

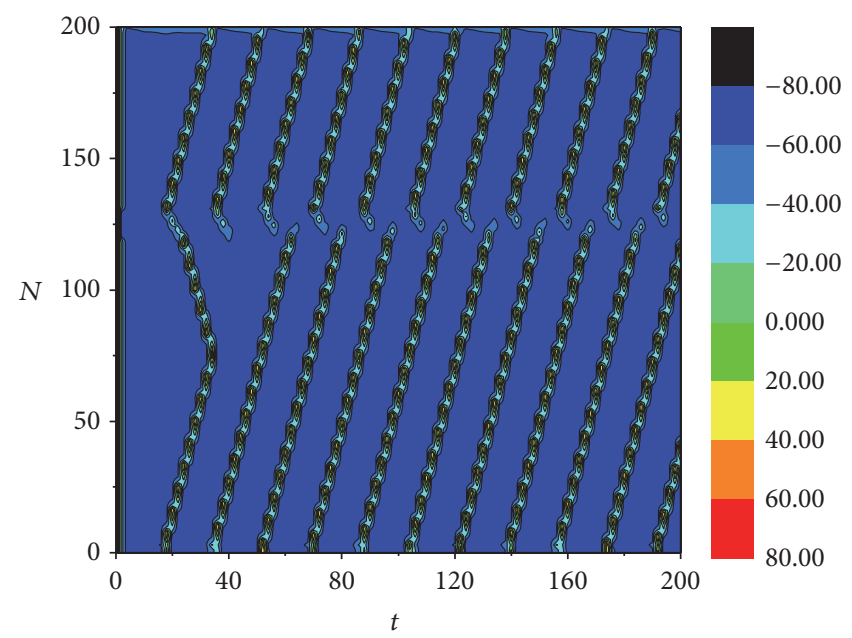

(d)

FIGURE 2: Wave propagation along axon when autapse is developed from node $i=3,131$ : external forcing is applied on node $i=3$; for time delays (a) $\tau=2$, (b) $\tau=10$, (c) $\tau=14$, and (d) $\tau=16$, the feedback gain in electric autapse is set as $g_{e}=1.5$ and external forcing $I_{\text {ext }}=20$ is imposed on node $i=3$. The injured area generated by decreasing the conductance of sodium from $G_{\mathrm{Na}}=120 \mathrm{mS} / \mathrm{cm}^{2}$ to $G_{\mathrm{Na}}=25.5 \mathrm{mS} / \mathrm{cm}^{2}$ : the axon is divided into 201 nodes and the injured area is marked from nodes $i=120$ to 130 . The snapshots are illustrated in color scale.

the autapse is formed, electrical autapse makes neuron give sensitive response to external forcing compared to the chemical autapse driving. In fact, this type of intrinsic time delay is finite and both of chemical and electrical autapse can cooperate with the signal processing and transmission for mixed signals such as time-varying stimuli on the neuron. The abnormality and injury on the axon can be described by ion channel blocking; for example, the conductance of ion channels is selected with diversity; the active channel numbers can be controlled by active agents or blocking agents.

In numerical studies, the active ratios $\chi_{\mathrm{Na}}$ and $\chi_{\mathrm{K}}$ can be selected with different values to describe the injury degree. Sampled action potentials besides the abnormality or heterogeneity can be recorded, and appropriate time delay and gain in the autapse can ensure the signal propagation and recovery because the blocked signals can be transmitted along the autapse. In the case of network, the autapse formation and driving can regulate the collective behaviors and prevent collapse of network $[59,60]$ by generating a pacemaker-like ordered wave. Extensive investigation asks for biological verification and detailed numerical studies. To present further description and understanding for this problem, numerical studies have been carried out. For simplicity, the conductance for sodium channels in local area is switched from $G_{\mathrm{Na}}=$ $120 \mathrm{mS} / \mathrm{cm}^{2}$ to $G_{\mathrm{Na}}=25.5 \mathrm{mS} / \mathrm{cm}^{2}$, the axon is divided into 201 nodes (the results are independent of the node number or length of axon, the length of axon is set as 100 space units and space step is 0.5 for numerical studies), the diffusion coefficient is set for $D=1.06$, membrane capacitance is selected by $C=1 \mu \mathrm{F} / \mathrm{cm}^{2}$, and injured area is selected between nodes $i=$ 120 and 130, and the autapse develops from node $i=3,131$ (or $i=3,135$ ). Firstly, the effect of electric autapse is investigated under different time delays and the results are plotted in Figure 2. 


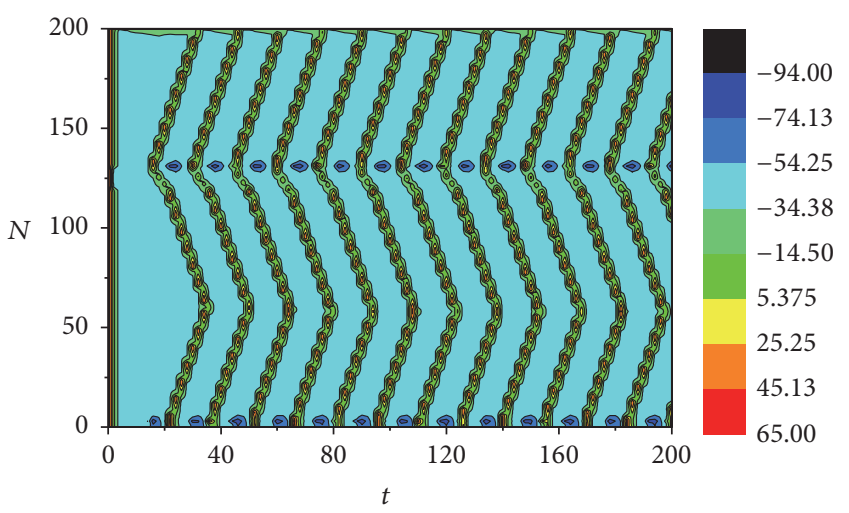

(a)

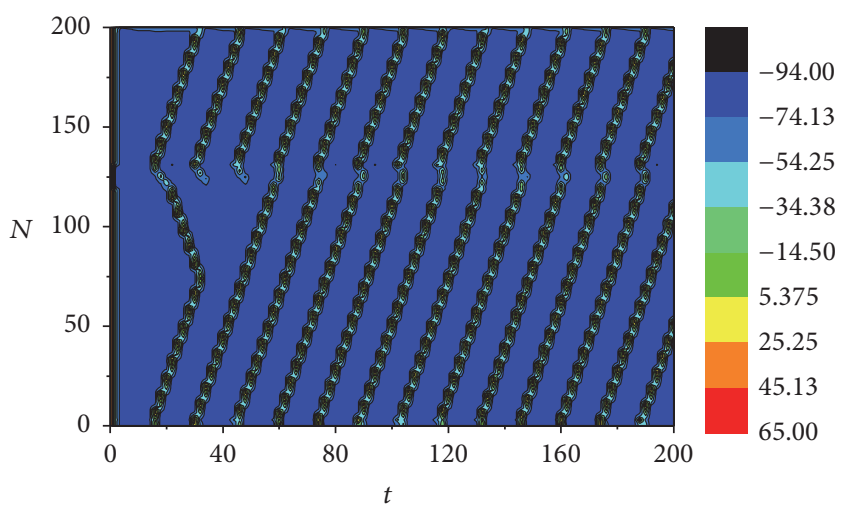

(c)

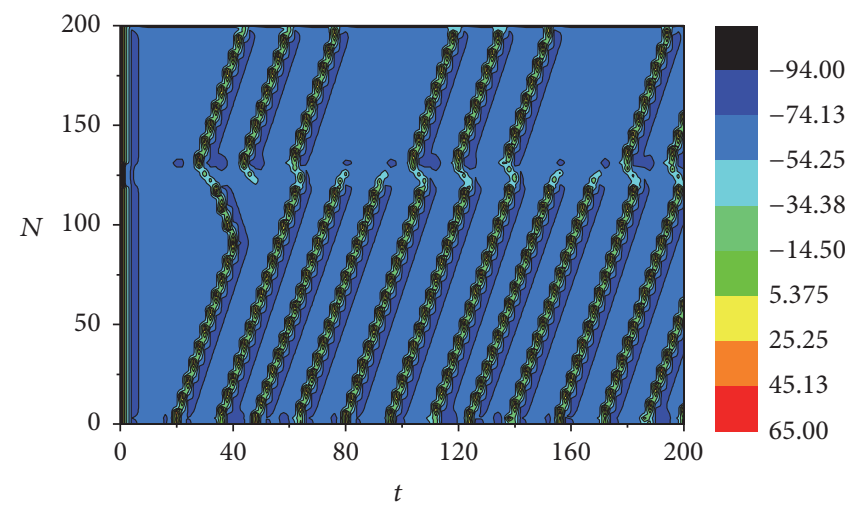

(b)

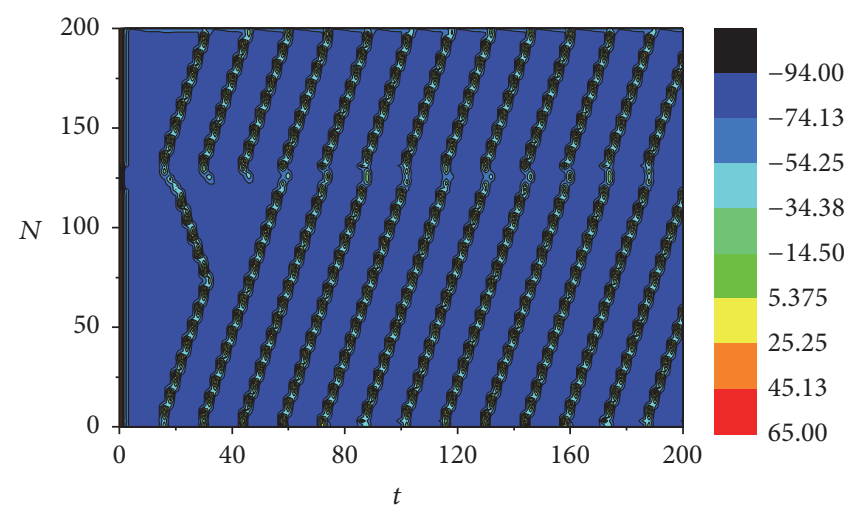

(d)

FIGURE 3: Wave propagation along axon when autapse is developed from node $i=3,131$, for gains (a) $g_{e}=-2$, (b) $g_{e}=-1,\left(\right.$ c) $g_{e}=1.5$, and (d) $g_{e}=10$; the time delay in electric autapse is set as $\tau=14$ and external forcing $I_{\text {ext }}=20$ is imposed on node $i=3$. The injured area generated by decreasing the conductance of sodium from $G_{\mathrm{Na}}=120 \mathrm{mS} / \mathrm{cm}^{2}$ to $G_{\mathrm{Na}}=25.5 \mathrm{mS} / \mathrm{cm}^{2}$ : the axon is divided into 201 nodes and the injured area is marked from nodes $i=120$ to 130 . The snapshots are illustrated in color scale.

It is confirmed that appropriate time delay in electric autapse is effective to propagate the blocked signal along the axon, and the information is kept complete by detecting the sampled time series. Extensive numerical results found that electric autapse can find appropriate time delay (e.g., $\tau=$ 14.5) to overcome the blocking and propagate the blocked signals; the sampled time series for membrane of nodes backward and forward the injured area can reach delayed synchronization completely. The effect of gains in electric autapse is also investigated, and the results are plotted in Figure 3.

It is found that the gain in the electric autapse also plays important role in enhancing signal propagating along the axon. The selection in gain can induce positive and negative feedback along the autapse loop, and negative feedback in electric autapse is helpful to propagate the blocked signal due to self-adaption, while positive feedback can generate new exciting source to further block the signal propagation. Surely, appropriate time delay in electric autapse can be effective to propagate signal and overcome the blocked area under positive feedback, and the results are plotted in Figure 4.

The results in Figure 4 confirmed that appropriate time delay can be found to set appropriate electric autapse so that the signal can be passed across the blocked or injured area.
That is to say, when autapse is formed, appropriate time delay and gain can be verified for the biological function of electric autapse. Furthermore, it is interesting to check the function of chemical autapse, and the external forcing current is still imposed on the node $i=3$. To discern and enhance the blocking effect on ion channels, inhibitory chemical autapse is investigated by setting $V_{\text {syn }}=-2$, and the results are plotted in Figure 5.

It is confirmed that large feedback gain in chemical autapse is helpful to propagate the signal across the injured area when appropriate time delay is applied on the chemical autapse. Furthermore, the feedback gain in chemical autapse is switched to negative value, and the results are plotted in Figure 6.

According to the results in Figures 5 and 6, the developed chemical autapse can be effective to help the blocked signal propagate across the injured area. We also checked the case for excitatory autapse; similar results are verified. Therefore, the formation and development of autapse connection enhance the self-adaption of neuron; thus signal can be propagated to overcome the blocking in the injured area.

Extensive numerical results confirmed that both of electric and chemical autapse (excitory and/or inhibitory) can 


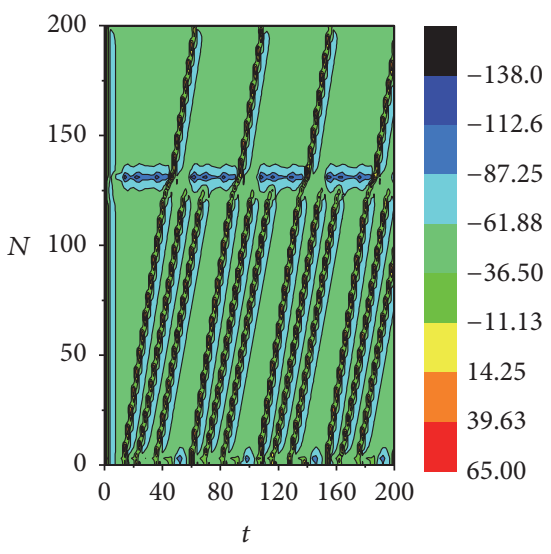

(a)

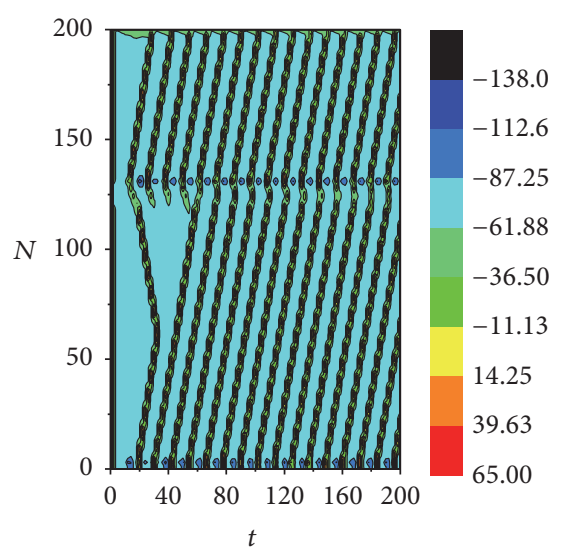

(b)

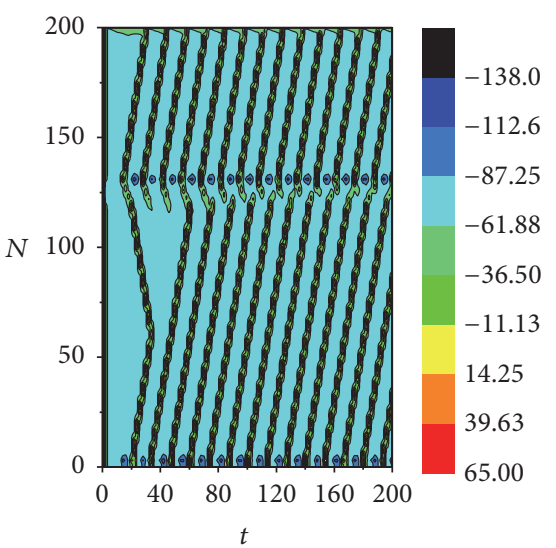

(c)

FIGURE 4: Wave propagation along axon when autapse is developed from node $i=3,135$, for time delays (a) $\tau=4$, (b) $\tau=11$, and (c) $\tau=14$ : the feedback gain in electric autapse is set as $g_{e}=-2.0$ and external forcing $I_{\text {ext }}=20$ is imposed on node $i=3$. The injured area generated by decreasing the conductance of sodium from $G_{\mathrm{Na}}=120 \mathrm{mS} / \mathrm{cm}^{2}$ to $G_{\mathrm{Na}}=25.5 \mathrm{mS} / \mathrm{cm}^{2}$ : the axon is divided into 201 nodes and the injured area is marked from nodes $i=120$ to 130 . The snapshots are illustrated in color scale.

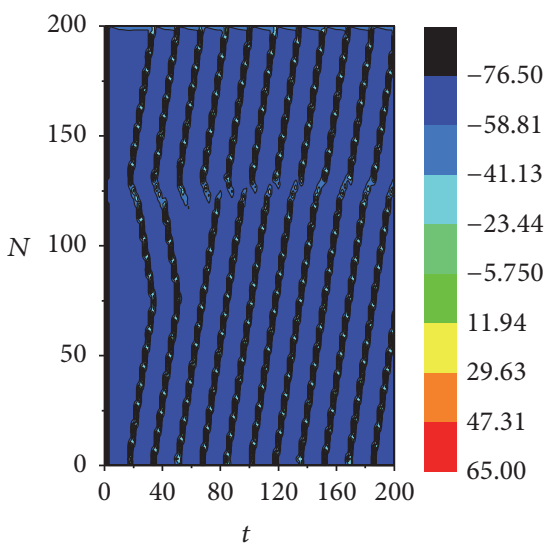

(a)

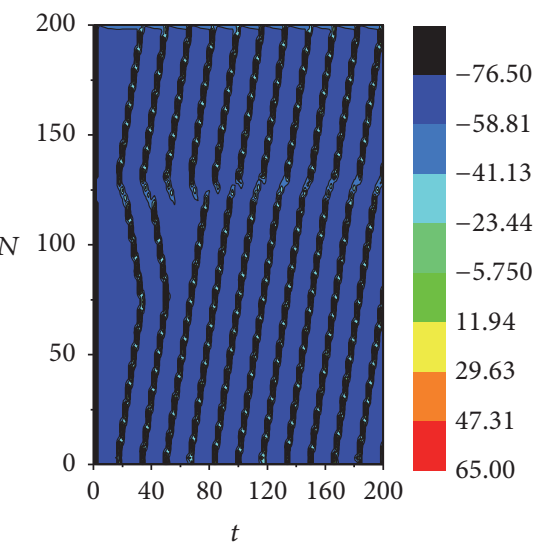

(b)

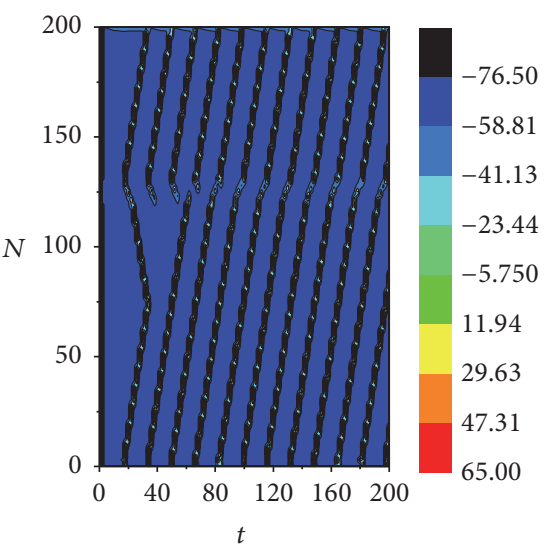

(c)

FIGURE 5: Wave propagation along axon when autapse is developed from node $i=3,135$, for gains (a) $g_{c}=10$, (b) $g_{c}=20$, and (c) $g_{c}=50$ : the time delay in electric autapse is set as $\tau=16$ and external forcing $I_{\text {ext }}=20$ is imposed on node $i=3$. The injured area generated by decreasing the conductance of sodium from $G_{\mathrm{Na}}=120 \mathrm{mS} / \mathrm{cm}^{2}$ to $G_{\mathrm{Na}}=25.5 \mathrm{mS} / \mathrm{cm}^{2}$ : inhibitor chemical autapse is used by setting $V_{\text {syn }}=-2$, the axon is divided into 201 nodes, and the injured area is marked from nodes $i=120$ to 130. The snapshots are illustrated in color scale.

confirm the biological function that signal can be propagated across the blocked area by applying appropriate time delay and feedback gain in the autapse. We also checked the case when some ion channels of potassium are blocked, and similar results are approached that appropriate time delay and gain in autapse are effective to reproduce (or restore) the blocked signals as well.

As mentioned above, synapse can be trigged close to the badly injured area and autapse can be formed to select appropriate time delay and gain, so that blocked signal (action potential or continuous wave) can be transmitted completely. The auxiliary loop for autapse can be effective to correct and restore the original signal successfully. In fact, our results just argue the possible formation mechanism and biological function for autapse in theoretical assumption and numerical verification. It is interesting for biological experts to check these results in experiments and detect possible formation of autapse connected to axon of neuron by carefully injuring such as poisoning in some ion channels of neuron.

\section{Conclusions}

The biological function and formation of autapse were discussed. We argued that the formation and development of autapse connection could result from the self-adaption and self-protection of neuron. Abnormal neurodevelopment or injury on the neuron (axon) makes blocking in signal transmission along the axon of neuron and signal communication between neurons. Following the abnormal area or heterogeneity, new synapse can be formed to connect the soma or dendrites connected to the soma, and autapse is generated by setting auxiliary loop via synapse connection. Switch between 


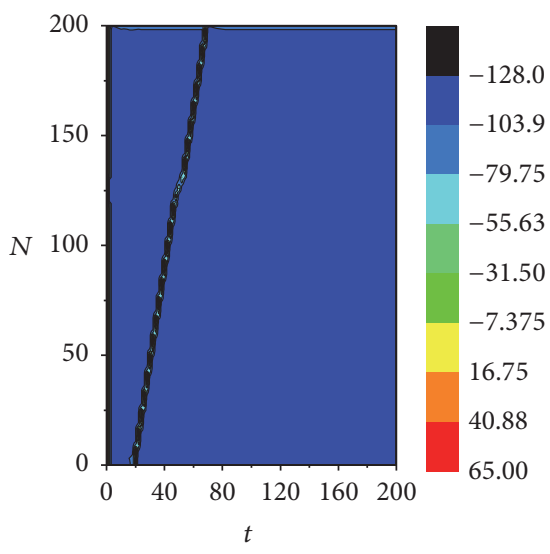

(a)

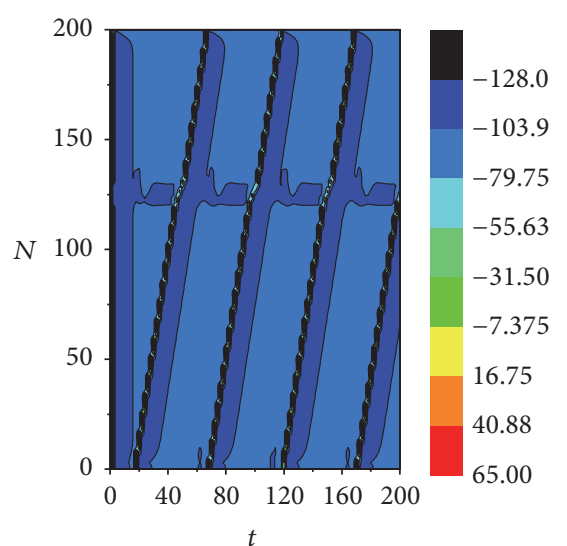

(b)

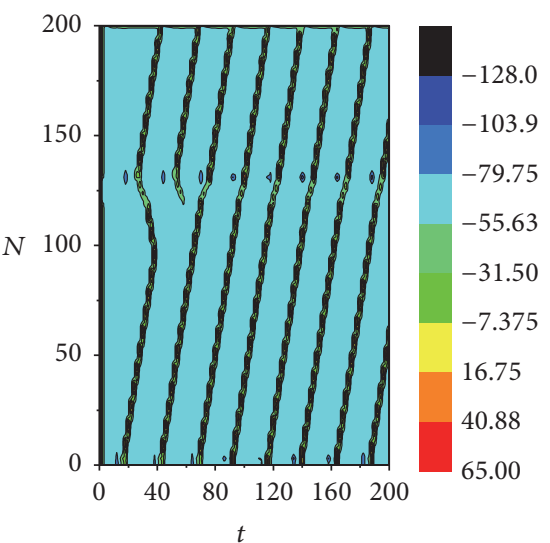

(c)

FIGURE 6: Wave propagation along axon when autapse is developed from node $i=3,135$, for gains (a) $g_{c}=-0.5$, (b) $g_{c}=-1$, and (c) $g_{c}=-2$ : the time delay in electric autapse is set as $\tau=10$ and external forcing $I_{\text {ext }}=20$ is imposed on node $i=3$. The injured area generated by decreasing the conductance of sodium from $G_{\mathrm{Na}}=120 \mathrm{mS} / \mathrm{cm}^{2}$ to $G_{\mathrm{Na}}=25.5 \mathrm{mS} / \mathrm{cm}^{2}$ : inhibitor chemical autapse is used by setting $V_{\text {syn }}=-2$, the axon is divided into 201 nodes, and the injured area is marked from nodes $i=120$ to 130 . The snapshots are illustrated in color scale.

positive and negative feedback in autapse is self-adaptive for mode selection in electrical activities; as a result, signal communication and transmission become available. These discussions could be helpful to understand the formation mechanism for autapse and its biological functions.

\section{Competing Interests}

The authors declare that they have no competing interests.

\section{Acknowledgments}

This work is partially supported by the National Natural Science Foundation of China under Grant nos. 11365014 and 11672122 .

\section{References}

[1] A. L. Hodgkin and A. F. Huxley, "A quantitative description of membrane current and its application to conduction and excitation in nerve," The Journal of Physiology, vol. 117, no. 4, pp. 500-544, 1952.

[2] E. M. Izhikevich, "Which model to use for cortical spiking neurons?" IEEE Transactions on Neural Networks, vol. 15, no. 5, pp. 1063-1070, 2004.

[3] J. L. Hindmarsh and R. M. Rose, "A model of the nerve impulse using two first-order differential equations," Nature, vol. 296, no. 5853, pp. 162-164, 1982.

[4] A. V. M. Herz, T. Gollisch, C. K. Machens, and D. Jaeger, "Modeling single-neuron dynamics and computations: a balance of detail and abstraction," Science, vol. 314, pp. 80-85, 2006.

[5] A. V. Chizhov, L. J. Graham, and A. A. Turbin, "Simulation of neural population dynamics with a refractory density approach and a conductance-based threshold neuron model," Neurocomputing, vol. 70, no. 1-3, pp. 252-262, 2006.

[6] H. G. Gu and B. B. Pan, "A four-dimensional neuronal model to describe the complex nonlinear dynamics observed in the firing patterns of a sciatic nerve chronic constriction injury model," Nonlinear Dynamics, vol. 81, no. 4, pp. 2107-2126, 2015.

[7] B. Ibarz, J. M. Casado, and M. A. F. Sanjuán, "Map-based models in neuronal dynamics," Physics Reports, vol. 501, no. 1-2, pp. 174, 2011.

[8] D. E. Postnov, L. S. Ryazanova, and O. V. Sosnovtseva, "Functional modeling of neural-glial interaction," BioSystems, vol. 89, no. 1-3, pp. 84-91, 2007.

[9] J. Tang, J.-M. Luo, and J. Ma, "Information transmission in a neuron-astrocyte coupled model," PLOS ONE, vol. 8, no. 11, Article ID e80324, 2013.

[10] M. Amiri, G. Montaseri, and F. Bahrami, "A phase plane analysis of neuron-astrocyte interactions," Neural Networks, vol. 44, pp. 157-165, 2013.

[11] M. Linne and T. O. Jalonen, "Astrocyte-Neuron Interactions: from experimental research-based models to translational medicine," in Computational Neuroscience, vol. 123 of Progress in Molecular Biology and Translational Science, pp. 191-217, Elsevier, 2014.

[12] V. Volman, M. Bazhenov, and T. J. Sejnowski, "Computational models of neuron-astrocyte interaction in epilepsy," Frontiers in Computational Neuroscience, vol. 6, article no. 58, 2012.

[13] R. Wang, I. Tsuda, and Z. Zhang, "A new work mechanism on neuronal activity," International Journal of Neural Systems, vol. 25, no. 3, Article ID 1450037, 2015.

[14] R. Wang and Y. Zhu, "Can the activities of the large scale cortical network be expressed by neural energy? A brief review," Cognitive Neurodynamics, vol. 10, no. 1, pp. 1-5, 2016.

[15] Y. Y. Xu, L. Ni, and R. B. Wang, "A neural network model of spontaneous up and down transitions," Nonlinear Dynamics, vol. 84, pp. 1541-1551, 2016.

[16] V. Volman, M. Perc, and M. Bazhenov, "Gap junctions and epileptic seizures-two sides of the same coin?" PLOS ONE, vol. 6, no. 5, Article ID e20572, 2011.

[17] B. Hille, Ion Channels of Excitable Membranes, Sinauer Associates, Sunderland, Mass, USA, 2001. 
[18] G. Schmid, I. Goychuk, and P. Hänggi, "Controlling the spiking activity in excitable membranes via poisoning," Physica, vol. 344, no. 3-4, pp. 665-670, 2004.

[19] M. Ozer, M. Perc, and M. Uzuntarla, "Controlling the spontaneous spiking regularity via channel blocking on NewmanWatts networks of Hodgkin-Huxley neurons," EPL, vol. 86, no. 4, Article ID 40008, 2009.

[20] J. Ma, A. Zhang, J. Tang, and W. Jin, "Collective behaviors of spiral waves in the networks of hodgkin-huxley neurons in presence of channel noise," Journal of Biological Systems, vol. 18, no. 1, pp. 243-259, 2010.

[21] J. Ma, Y. Wu, H. Ying, and Y. Jia, "Channel noise-induced phase transition of spiral wave in networks of Hodgkin-Huxley neurons," Chinese Science Bulletin, vol. 56, no. 2, pp. 151-157, 2011.

[22] L. Huang, J. Ma, J. Tang, and F. Li, “Transition of ordered waves in neuronal network induced by diffusive poisoning of ion channels," Journal of Biological Systems, vol. 21, no. 1, Article ID 1350002, 2013.

[23] X. Sun and X. Shi, "Effects of channel blocks on the spiking regularity in clustered neuronal networks," Science China Technological Sciences, vol. 57, no. 5, pp. 879-884, 2014.

[24] M. Lv and J. Ma, "Multiple modes of electrical activities in a new neuron model under electromagnetic radiation," Neurocomputing, vol. 205, pp. 375-381, 2016.

[25] M. Lv, C. Wang, G. Ren, J. Ma, and X. Song, "Model of electrical activity in a neuron under magnetic flow effect," Nonlinear Dynamics, vol. 85, no. 3, pp. 1479-1490, 2016.

[26] J. Ma and J. Tang, "A review for dynamics of collective behaviors of network of neurons," Science China Technological Sciences, vol. 58, no. 12, pp. 2038-2045, 2015.

[27] J. Ma, H. Qin, X. Song, and R. Chu, "Pattern selection in neuronal network driven by electric autapses with diversity in time delays," International Journal of Modern Physics B, vol. 29, no. 1, Article ID 1450239, 2015.

[28] H. X. Qin, Y. Wu, C. N. Wang, and J. Ma, "Emitting waves from defects in network with autapses," Communications in Nonlinear Science and Numerical Simulation, vol. 23, no. 1-3, pp. 164-174, 2015.

[29] M. Ozer, M. Uzuntarla, T. Kayikcioglu, and L. J. Graham, “Collective temporal coherence for subthreshold signal encoding on a stochastic small-world Hodgkin-Huxley neuronal network," Physics Letters A, vol. 372, no. 43, pp. 6498-6503, 2008.

[30] R. Uzun, M. Ozer, and M. Perc, "Can scale-freeness offset delayed signal detection in neuronal networks?” EPL, vol. 105, no. 6, Article ID 60002, 2014.

[31] J. M. Bekkers, "Synaptic transmission: functional autapses in the cortex," Current Biology, vol. 13, no. 11, pp. R433-R435, 2003.

[32] R. Saada, N. Miller, I. Hurwitz, and A. J. Susswein, "Autaptic excitation elicits persistent activity and a plateau potential in a neuron of known behavioral function," Current Biology, vol. 19, no. 6, pp. 479-484, 2009.

[33] L. Wang and Y. Zeng, "Control of bursting behavior in neurons by autaptic modulation," Neurological Sciences, vol. 34, no. 11, pp. 1977-1984, 2013.

[34] K. Suga, "Isoproterenol facilitates GABAergic autapses in fastspiking cells of rat insular cortex," Journal of Oral Science, vol. 56, no. 1, pp. 41-47, 2014.

[35] D. Guo, M. Chen, M. Perc et al., "Firing regulation of fastspiking interneurons by autaptic inhibition," EPL, vol. 114, no. 3, 2016 .
[36] A. B. Karabelas and D. P. Purrura, "Evidence for autapses in the substantia nigra," Brain Research, vol. 200, no. 2, pp. 467-473, 1980.

[37] M. S. Goldman, "Memory without Feedback in a Neural Network," Neuron, vol. 61, no. 4, pp. 621-634, 2009.

[38] J. M. Bekkers and C. F. Stevens, "Excitatory and inhibitory autaptic currents in isolated hippocampal neurons maintained in cell culture," Proceedings of the National Academy of Sciences, vol. 88, no. 17, pp. 7834-7838, 1991.

[39] W.-X. Shi and S. Rayport, "GABA synapses formed in vitro by local axon collaterals of nucleus accumbens neurons," The Journal of Neuroscience, vol. 14, no. 7, pp. 4548-4560, 1994.

[40] H. Van Der Loos and E. M. Glaser, "Autapses in neocortex cerebri: synapses between a pyramidal cell's axon and its own dendrites," Brain Research, vol. 48, pp. 355-360, 1972.

[41] X. L. Song, C. N. Wang, J. Ma, and J. Tang, "Transition of electric activity of neurons induced by chemical and electric autapses," Science China Technological Sciences, vol. 58, no. 6, pp. 10071014, 2015.

[42] E. Yilmaz and M. Ozer, "Delayed feedback and detection of weak periodic signals in a stochastic Hodgkin-Huxley neuron," Physica A: Statistical Mechanics and Its Applications, vol. 421, pp. 455-462, 2015.

[43] E. Yilmaz, V. Baysal, M. Perc, and M. Ozer, "Enhancement of pacemaker induced stochastic resonance by an autapse in a scale-free neuronal network," Science China Technological Sciences, vol. 59, no. 3, pp. 364-370, 2016.

[44] E. Yilmaz, V. Baysal, M. Ozer, and M. Perc, "Autaptic pacemaker mediated propagation of weak rhythmic activity across smallworld neuronal networks," Physica A: Statistical Mechanics and its Applications, vol. 444, pp. 538-546, 2016.

[45] H.-T. Wang and Y. Chen, "Firing dynamics of an autaptic neuron," Chinese Physics B, vol. 24, no. 12, Article ID 128709, 2015.

[46] Q. Wang, Y. B. Gong, and Y. N. Wu, "Autaptic self-feedbackinduced synchronization transitions in Newman-Watts neuronal network with time delays," The European Physical Journal $B$, vol. 88, article 103, 2015.

[47] Y. Wu, Y. Gong, and Q. Wang, "Autaptic activity-induced synchronization transitions in Newman-Watts network of Hodgkin-Huxley neurons," Chaos, vol. 25, no. 4, Article ID 043113, 2015.

[48] H. Qin, J. Ma, C. Wang, and R. Chu, "Autapse-induced target wave, spiral wave in regular network of neurons," Science China: Physics, Mechanics and Astronomy, vol. 57, no. 10, pp. 1918-1926, 2014.

[49] J. Ma, X. Song, W. Jin, and C. Wang, "Autapse-induced synchronization in a coupled neuronal network," Chaos, Solitons and Fractals, vol. 80, pp. 31-38, 2015.

[50] J. Ma, X. Song, J. Tang, and C. Wang, "Wave emitting and propagation induced by autapse in a forward feedback neuronal network," Neurocomputing, vol. 167, pp. 378-389, 2015.

[51] Y. Fan, F. Xu, G. Huang, T. J. Lu, and W. Xing, "Single neuron capture and axonal development in three-dimensional microscale hydrogels," Lab on a Chip, vol. 12, no. 22, pp. 47244731, 2012.

[52] C. S. Herrmann and A. Klaus, "Autapse turns neuron into oscillator," International Journal of Bifurcation and Chaos in Applied Sciences and Engineering, vol. 14, no. 2, pp. 623-633, 2004. 
[53] G. D. Ren, G. Wu, J. Ma, and Y. Chen, "Simulation of electric activity of neuron by setting up a reliable neuronal circuit driven by electric autapse," Acta Physica Sinica, vol. 64, no. 5, Article ID 058702, 2015.

[54] Y. Li, G. Schmid, P. Hänggi, and L. Schimansky-Geier, "Spontaneous spiking in an autaptic Hodgkin-Huxley setup," Physical Review E - Statistical, Nonlinear, and Soft Matter Physics, vol. 82, no. 6, Article ID 061907, 2010.

[55] H. T. Wang, L. F. Wang, Y. L. Chen et al., "Effect of autaptic activity on the response of a Hodgkin-Huxley neuron," Chaos, vol. 24, Article ID 033122, 2014.

[56] G. Schmid, I. Goychuk, and P. Hänggi, "Effect of channel block on the spiking activity of excitable membranes in a stochastic Hodgkin-Huxley model," Physical Biology, vol. 1, no. 2, pp. 6166, 2004.

[57] G. Schmid, I. Goychuk, and P. Hänggi, "Stochastic resonance as a collective property of ion channel assemblies," Europhysics Letters, vol. 56, no. 1, pp. 22-28, 2001.

[58] P. Jung and J. W. Shuai, "Optimal sizes of ion channel clusters," Europhysics Letters, vol. 56, no. 1, pp. 29-35, 2001.

[59] X. Song, C. Wang, J. Ma, and G. Ren, "Collapse of ordered spatial pattern in neuronal network," Physica A, vol. 451, pp. 95$112,2016$.

[60] J. Ma, Y. Xu, G. Ren, and C. Wang, "Prediction for breakup of spiral wave in a regular neuronal network," Nonlinear Dynamics, vol. 84, no. 2, pp. 497-509, 2016. 


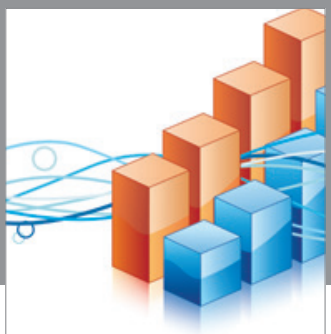

Advances in

Operations Research

vatem alat4

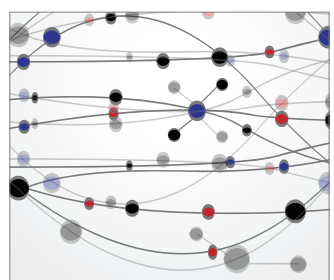

\section{The Scientific} World Journal
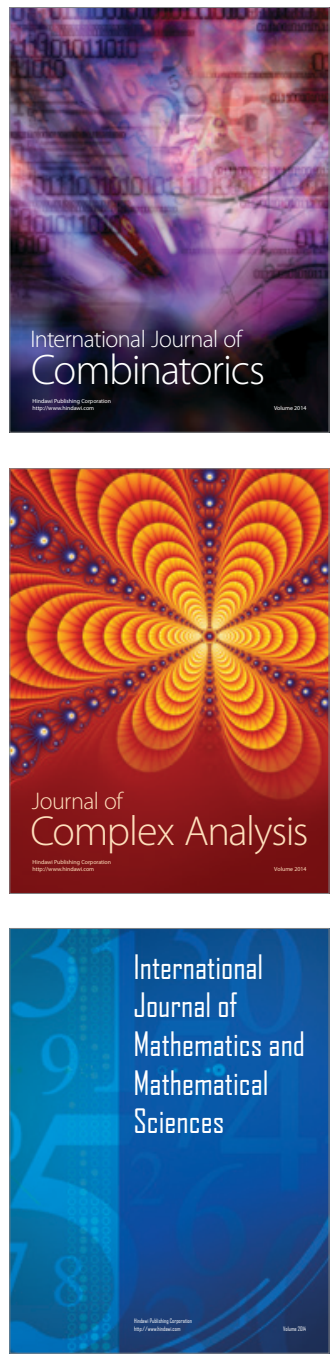
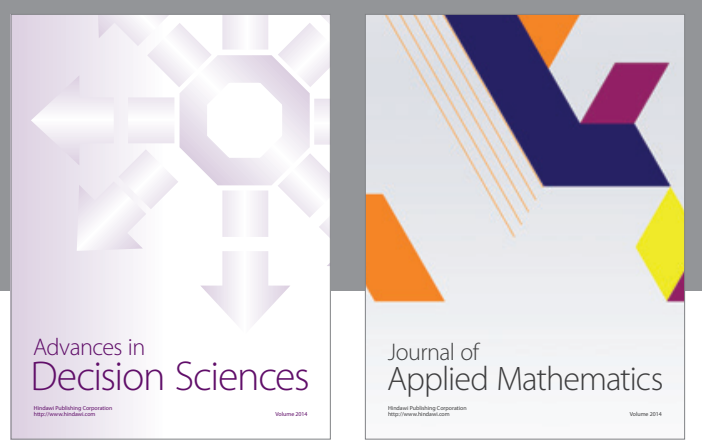

Algebra

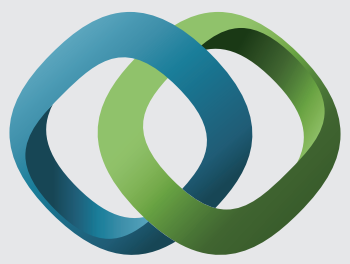

\section{Hindawi}

Submit your manuscripts at

https://www.hindawi.com
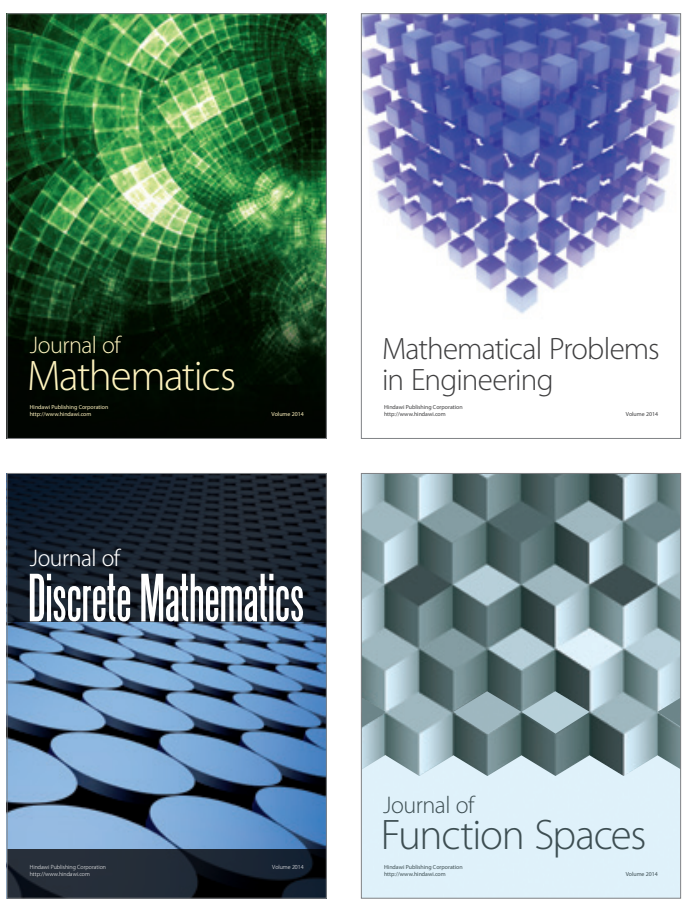

Mathematical Problems in Engineering
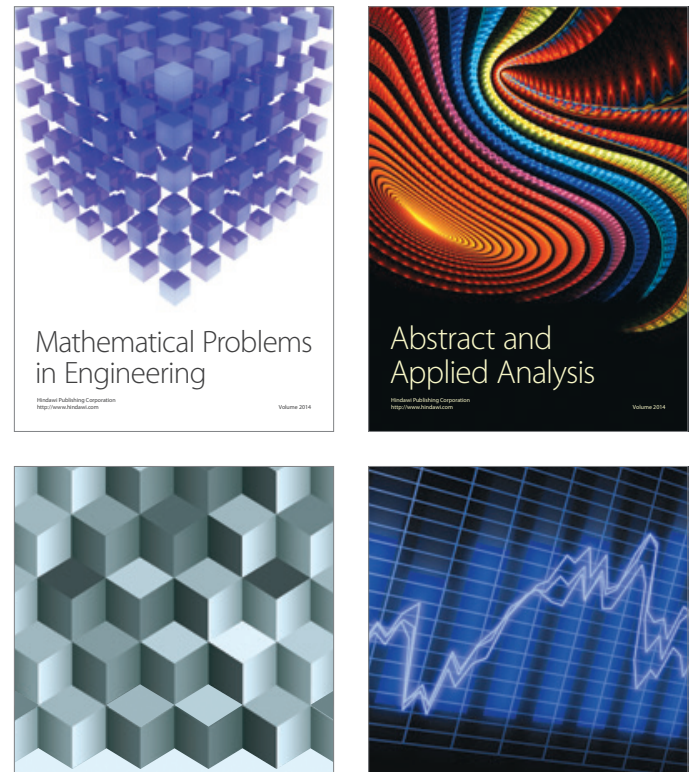

Journal of

Function Spaces

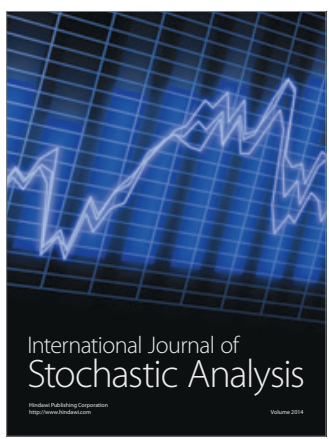

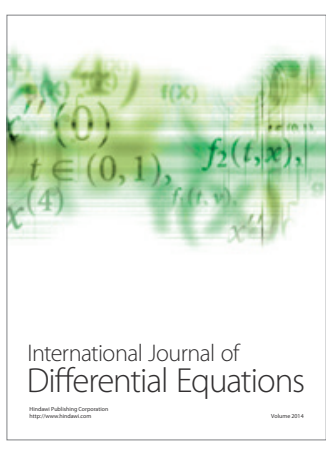
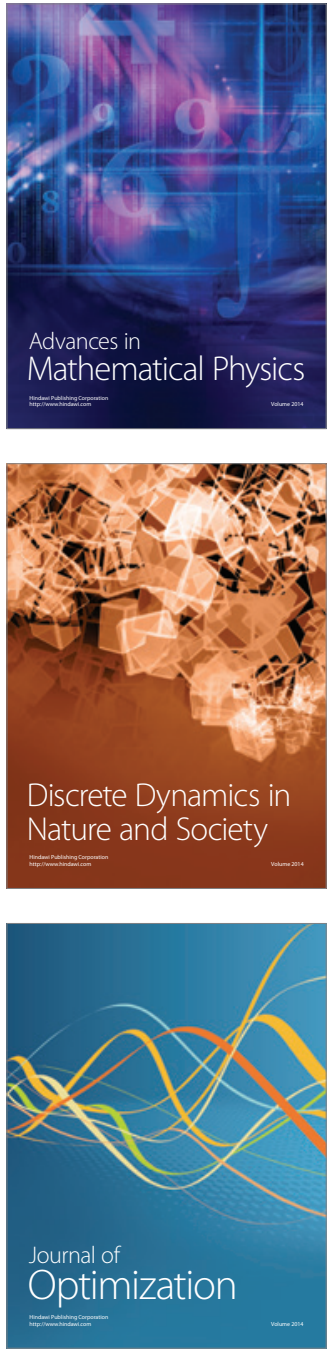